\title{
Research Directions on Diffusion and Adoption of Information Technology
}

\author{
Karlheinz Kautz \\ Norwegian Computing Center \\ P.O. Box 114 Blindern, N-0314 Oslo, Norway, \\ Email:Karl.Kautz@nr.no
}

Jan Pries-Heje
Institute of Informatics and Management Accounting,
Copenhagen Business School
Howitzvej 60,DK-2000 Frederiksberg, Denmark,Email:dsjph@cbs.dk

The IFIP WG 8.6 on diffusion, transfer, and implementation of information technology has held its first Working Conference on the theme of Adoption and Diffusion of IT at Leangkollen, Oslo in October 1995. In the working group the term information technology is used in its broadest sense spanning from traditional information systems, over modern communication technology to best practice routines in system development and software engineering.

Information technology is playing an increasingly important role in many organisations, sectors and countries. The adoption and diffusion process takes place between one country and another, between research and development organisations, between commercial organisations and the open marked in general, as well as between different departments in one and the same organisation. However, there are still many problems in the practical adoption of the technology, and not often are the results expected by the use of IT achieved. This may be, among other reasons, due to inappropriate attention to the adoption process and associated change processes in communities aiming at the utilization of the technology.

This volume contains twelve contributions which reflect different aspects of diffusion and adoption of information technology. As such, they give an initial overview over the diversity of work done in a subject area which implicitly has been a part of IT research for 20 years, but which only recently has gained attention as a field of interest on its own.

When reviewing the contributions one major distinction immediately arises. Most of the work considers adoption and diffusion within or into organisations, either companies or public organisations and only few papers take another viewpoint, namely that of looking at adoption and diffusion either in sectors or between nations.

In total we have identified five lines of work. The first aims at identifying or specifying overall frameworks and models for the whole diffusion and adoption process. The second group deals with adoption and diffusion within organisations in a rather normative way. Although having sound theoretical and analytical basis, the main message is normative; recommendations are given to planners, change agents, managers etc. on how to make diffusion and adoption happen in an organisation.

Representatives of the third direction are not focusing on the whole process, but on certain selected adoption and diffusion issues. They take a mainly theoretical and analytical 
perspective, but the research methods used are quite different. One part of this work uses qualitative methods, such as case-studies, to make in-depth explorations and identification of important diffusion and adoption issues. The other part which we see as an group uses quantitative methods focusing on measuring and modelling the adoption and diffusion process.

In the following a brief summary of all contributions will be provided.

\section{Frameworks and models}

General models and frameworks are used to predict the course of a diffusion and adoption process. The changes of success for such an endeavour are one important part of research within the field of technology transfer.

Jaakkola discusses a collection of quantitative mathematical diffusion models which are used to describe the diffusion of a product by forecasting the number of potential adopters and users, and (if quantifiable) the benefits that the adopter community might gain from the technology. A major problem with mathematical models is that they do not cover the variables which describe the context of the diffusion. Another problem is that most of them assume a stable environment. Using the case of diffusion of mobile telephones Jaakkola shows one general predicament of the mathematical approach, namely that it often leads to a too optimistic prediction in the short term run and a too pessimistic one in a long term. However, it is argued that mathematical models are informative in a less precise way and that they can be a valuable supplement for other diffusion prediction approaches.

Leon proposes such a approach. However, his framework aims not at forecasting the success of a technology in general, but is directed towards the diffusion of formal software development methods in software organisations. He suggests the use of so-called adoption profiles to analyse an organisation's conditions for adopting a technology. A profile consists of contextual aspects like pressures which influence the transfer process, the formality of transfer processes, the adopters' attitudes and interdependency between providers and receptors; and technological aspects, like the adaptability, the maturity, the impact on work procedures, and the differences from technology already in use. Adoption profiles are then used to plan and choose actions to minimise the risk of failure when introducing technology. The value of the approach is demonstrated with two case studies.

\section{Normative recommendations}

From a sound theoretical basis two contributions present a very strong normative bias that offers advice and recommendations to change agents, managers and others who are engaged in technology transfer.

A proposal which results in technical support for transfer activities is based on the idea that technology introduction has to be based on a systematic process. The problem for tool support lies in identifying the replicable parts. Comparetta, Fowler, Juristo and Levine present the prototype of a software tool and the base model that evolved during the tool' s development process. The base model distinguishes three phases of technology transfer. These are planning and purchase, implementation and sustaining use. Eight key activities are supported by the tool: establish team, define desired process, establish current state, identify gap, develop solutions, pilot use of technology and evaluate result, roll out and ongoing use, wrap up and shut down transfer team. The prototype helps gather information about the starting conditions of a transfer project and supports the generation of an adoption plan by taking into account the tasks and sub- 
tasks of a transfer endeavour.

A different implementation planning model is proposed by McMaster and Vidgen. Again, also these authors stress the need for a planned project. However, they see implementation as a vital part of development. In contrast to Lien this means that implementation has to be taken into account from the very beginning of development work. The methods they use to plan the activities to handle the elusive, and partly unpredictable factors of human, not technical concern, are borrowed from the marketing research discipline. The key activity in their model is to take into account the needs of all involved stake holder groups. This means that, first of all, stakeholder groups have to be identified, then their interests have to be analysed, and the assumptions made by the analysts have to be compared in a gap analysis to the stakeholders' attitudes. As said before, interview techniques from marketing are proposed to perform these tasks. On this background the other crucial element of their model is developed: That is a communication strategy which will aid in explaining the introduction process to all involved. The benefits of such a strategy are demonstrated by a particular case, the introduction of a computer-based access control system for a car park.

\section{Organisational Issues}

Three contributions focus on one or more specific issues of the adoption and diffusion process. These specific issues which are viewed as being of major significance are very diverse, they span from learning to culture and politics.

Thoresen claims that organisational adoption of IT systems requires distribution, sharing and pooling of knowledge. To say it another way, successful adoption requires "Learning at work". Thoresen looks at how learning takes place in organisations, what resources are used, and what are the elements that can facilitate or hinder learning and thereby adoption of IT? Based on a qualitative interview-study and grounded theory analysis, she identifies several resources for learning. E.g. overlapping skills, expert users, meeting places, and continually overlapping groups. She also identifies several hindrances for successful learning/adoption such as division of work enforced by organisational boundaries, peripheral participation brought about by work pressure, IT-complexity, and the varying degree of IT-usefulness for different groups.

Kautz also looks at organisational adoption of IT. However, Kautz uses an interpretive framework to understand what can influence the adoption and diffusion process. The framework focuses on three issues: (1) The content of the IT being introduced. (2) The context in which the introduction takes place, and (3) the cultural and political processes taking place as part of the introduction of IT in an organisation. Kautz uses the framework in a case-study on the introduction of email in a public sector organisation. Based on interviews and studies of documents he concludes that the use of the interpretive framework broadens the analysis that is needed before the introduction of IT. That has some value in itself because it may help minimize the risk of adoption-failure.

Hughes, Kristoffersen, O'Brien and Rouncefield take up the issue of organisational politics that were also in Kautz's framework. Using two case-studies of the introduction of video-based communication systems Hughes, Kristoffersen, O'Brien and Rouncefield find that organisational politics on the one hand can drive and encourage the exploration of new IT, but on the other hand (and at the same time) foster ways of organizing and managing work that conflicts with the adoption and implementation of the new IT. 


\section{Modelling and Measuring}

Whereas a strong qualitative tendency characterised the papers presented so far, three other articles are grounded in a quantitative research tradition as they use quantitative modelling and measuring to explain adoption and diffusion processes.

Watad and Ospina uses data from 140 public organisations to focus on the link between context and change enabled by new IT. They conclude that neither IT nor strategic choice alone control the change process. Instead, they say, change is moderated by factors linked to the organisational setting. Factors like structure, tasks and goals. Watad and Ospina also claim that their findings support the so-called emergent perspective of organisational change. Based on their findings Watad and Ospina recommend that managers responsible for adoption and diffusion changes have to take into account the relation between human and technical aspects and focus simultaneously on external demands and the internal meeting of those demands.

Closely related to the Watad and Ospina paper is the paper by Thong and Yap. They present a factor-based study of the relation between IT adoption and contextual factors. Contextual factors taken into account are CEO innovativeness, information intensity, adoption attitudes, IT knowledge, and competition. Thong and Yap sent out a questionnaire to 1200 small Singapore businesses, where "small" mean businesses with fixed assets less than 7.2 M US\$, less than 100 employees, and annual sales less than 9 M US\$. Thong and Yap got 172 (16\%) completed questionnaires back. The analysis revealed that the extent of IT adoption is mainly determined by the level of IT knowledge. Furthermore it was shown that small businesses are more likely to adopt new IT when they have an innovative CEO and a positive adoption attitude. However, these two factors do not affect the extent of subsequent IT adoption.

Moore and Benbasat do not focus on the organisational level but on individual level. They ask the question: What are the factors influencing individual decisions to use IT? To answer that question, Moore and Benbasat distributed 810 questionnaires to users and non-users of personal work stations in 7 organisations, and got a $67 \%$ response rate. The questionnaire tested a research model with four components (usage, attitude towards adopting, subjective norm, and voluntariness), each with 1-8 dimensions. E.g. the component Usage had two dimensions: (1) Diversity of use. (2) Intensity. The research model was developed on the basis of Reasoned Action and Diffusion of Innovations theory. Moore and Benbasat conclude, based on statistical analysis, that the research model can be used for understanding the utilization of IT. Furthermore, in consistency with results from diffusion research, they also find that the most significant perceptions that had an effect on degree of use were: (1) ease of use, (2) relative advantage, and (3) compatibility.

\section{Diffusion and adoption within sectors and nations}

The adoption and diffusion on a regional, national, or sectorial level builds the focus of the fifth identified group of contributions.

Robertson, Swan, and Newell focus on decision making processes regarding the introduction of information technology. They are concerned with the diffusion of computer aided production management technology in a particular industry sector, the automotive sector in the United Kingdom. Attention is given to the influence of interorganisational networks on the decision making process. The article includes a disapproving critique of Roger's fundamental diffusion model because he describes diffusion as a simple, linear, sequential process, driven by the needs of potential adopters. This does not adequately address the dynamics of the relationship between suppliers and adopters and the active participation of the potential adopters in the 
process. The authors have analysed three cases and explain how awareness has been developed and which social networks informed the decisions. A typical network for information collection consist of free seminars organized by professional associations and performed by vendors and consultants, university courses on the subject, and support by commercial consultants. Especially important is the role of consultants as boundary spanners between the networks. Another result is that diffusion is often driven by fashion, rather than by an efficient choice perspective. The drive leads to a situation where inappropriate technology is adopted as the result of the knowledge selectively accessed by the decision makers.

The comparison of models used for strategic information technology decision making in two different regions, the Chinese and the Western world is the subject of Elliot's submission. He has selected one business sector, the banking business to investigate the decision making processes employed when considering the introduction of computer technology. On the basis of four cases Elliot concludes that there are common factors in these decisions. The business needs and requirements are especially important but the Commissioners of Banking's requirement also influences the decision to utilise or not to utilise information technology by Chinese banks. The same is true for the core processes in decision making. These comprise requirements' determination, capacity monitoring, proposal, and consideration and approval by CEOs. The final outcome of the study is that the differences between Western and Chinese decision making are not that big, at least not in the banking sector. The elucidated factors and processes in China are substantially similar to those used in Western organisations.

Together the contributions serve as an entry to the subject of diffusion and adoption of information technology, a subject in which further research results with the emergence of new technologies can frequently be expected 